\title{
Full Series Algorithm of Automatic Building Extraction and Modelling From LiDAR Data
}

\author{
Fayez Tarsha Kurdi*, Zahra Gharineiat and Glenn Campbell \\ School of Civil Engineering \& Surveying, Faculty of Health, \\ Engineering and Sciences, University of Southern Queensland, \\ Springfield QLD 4350, Australia \\ \{fayez.tarshakurdi, zahra.gharineiat, \\ glenn.campbell\}@usq.edu.au
}

\author{
Emon Kumar Dey and Mohammad Awrangjeb \\ Institute of Integrated and Intelligent Systems \\ Griffith University, Nathan QLD 4111, Australia \\ emonkumar.dey@griffithuni.edu.au; \\ m.awrangjeb@griffith.edu.au
}

\begin{abstract}
This paper suggests an algorithm that automatically links the automatic building classification and modelling algorithms. To make this connection, the suggested algorithm applies two filters to the building classification results that enable processing of the failed cases of the classification algorithm. In this context, it filters the noisy terrain class and analyses the remaining points to detect missing buildings. Moreover, it filters the detected building to eliminate all undesirable points such as those associated with trees overhanging the building roof, the surrounding terrain and the façade points. In the modelling algorithm, the error map matrix is analysed to recognize the failed cases of the building modelling algorithm with these buildings being modelled with flat roofs. Finally, the region growing algorithm is applied on the building mask to detect each building and pass it to the modelling algorithm. The accuracy analysis of the classification and modelling algorithm within the global algorithm shows it to be highly effective. Hence, the total error of the building classification algorithm is $0.01 \%$ and only one building in the sample dataset is rejected by the modelling algorithm and even that is modelled, but with a flat roof. Most of the buildings have Segmentation Accuracy and Quality factor less than 5\% (error less than $5 \%$ ) which means that the resulting evaluation is excellent.
\end{abstract}

Keywords-LiDAR, Classification, Modelling, Filtering, Segmentation

\section{INTRODUCTION}

Aircraft equipped with modern Light Detection And Ranging (LiDAR) systems measure precise elevation information. By emitting a huge number of laser pulses per second $(100,000$ or more) onto the Earth's surface they take measurements at resolutions exceeding one point per square meter [26]. For each laser pulse, the orientation angles, the return distance, and the origin coordinates are measured through the components of the scanning system such as the Global Position System (GPS) and the Inertial Measurement Unit (IMU) [27]. Furthermore, the full waveform, the laser intensity and Red, Green and Blue (RGB) values are saved for each measured point. The ASCII format files is not suitable to record the resulting enormous data volume, that is why a simple binary exchange file format (LAS) is suggested by the American Society for Photogrammetry and Remote Sensing (ASPRS). This format still represents an immense volume and needs to be compressed. To facilitate this, the compressed file format (LAZ) has become prevalent [25]. The result of these massive number of measurements is a dense $3 \mathrm{D}$ point cloud.

The outcome of airborne laser scanning is a $3 \mathrm{D}$ point cloud. This point cloud provides the input data for a series of operations that aims to classify and then model the points into their appropriate classes such as terrain, buildings and vegetation [21]. In literature, from 1995 until the present, hundreds of research papers suggested automatic approaches for achieving the automatic LiDAR data classification and modelling [28].

The construction of a full series algorithm that links the automatic classification and modelling algorithms must overcome several obstacles such as misclassification cases and the failure of building modelling. These failures break the series of automatic processing and / or generate unreliable results.

The novelty and technical contribution of this work may be summarised as follows. First, the suggestion of a global algorithm that links any two automatic classification and modelling algorithms. Second, this algorithm corrects the misclassification cases such as the missing buildings and the miss-classified non-building objects. Third, it considers also cases of building modelling failure. Finally, the application of this algorithm provides a city model directly after scanning in addition to the point cloud.

\section{BACKGROUND}

Building classification and modelling have various practical applications. Accurate modelling of the extracted building from a LiDAR point cloud data can help to create accurate city model, computer graphics, gaming, reverse engineering, urban mapping, and solar potential estimation [1, $2,3]$. Processing a raw LiDAR point cloud data to the final 3D modelling of buildings can be divided into three major steps. Firstly, detection and removal of outliers from the raw point cloud [2]. Secondly, separation of building points (e.g., roof, wall, and façade points) from the noise-free point cloud [4]. Finally, generation of 3D building models from the extracted building points $[5,21]$.

Outliers can be observed in point cloud data for several reasons, including multiple reflections, occlusions, and limitations of the data collecting aircraft [1]. Even after separating the building points, outliers can be observed [6]. Outliers degrade the performance of the building reconstruction techniques [2]. Both unsupervised and supervised approaches of outlier detection from point cloud data have been using by several authors for accurate building extraction and modelling in the literature $[2,6,7,8]$. The unsupervised approaches mainly use statistical formulas, distance and density-based techniques [8], whereas, the supervised approaches bespoke features to train the conventional machine learning classifiers [7]. However, because of the absence of properly labelled ground truth, supervised approaches of outlier detections are difficult to realize in practice $[7,8]$. 
The task of building point segmentation/extraction from the outlier-free LiDAR point cloud data can be divided into three major steps $[9,10]$. Filtering based on an elevation threshold to divide the point cloud data into terrain and nonterrain groups is the initial step [6]. Next, the segmentation of the non-terrain points into several planar candidate building parts. Different plane fitting algorithms (e.g., Principal Component Analysis (PCA) RANdom SAmple Consensus (RANSAC), and their variations) are commonly used in this stage $[2,11]$. Finally, to remove spurious planes and refine the actual roof planes, several post-processing steps are applied by different authors $[4,12]$. Machine learning approaches also have been used for building area segmentation $[6,14,15]$. In this case, most of the approaches classify the input point cloud into classes, such as, buildings, trees, vehicles, and terrain. Extracted buildings are then used for several purposes, e.g., city modelling [6], change detection [5], solar potential estimation [16]. In most cases handcrafted geometric and color features are used to train the conventional classifiers Random Forest (RF) and Support Vector Machine (SVM). Recently deep learning-based approaches have become popular to classify building point clouds from the raw input point cloud data $[17,18,19,20]$.

Existing approaches to the reconstruction of individual buildings from the extracted building point cloud data can be mainly categorised into model-driven and data-driven approaches [12]. The former is also known as parametric or bottom-up approaches and the latter is also known as a nonparametric or top-down approaches [11]. A predefined database of parameterised building models must be first constructed and then the best fitting model for each building in the input LiDAR point cloud is chosen. Thus, this approach is highly dependent on the stored predefined database, and for any unspecified or complex building structure, it fails if no model exists in the stored library. The data-driven approach, on the other hand, do not depend on the primitive shapes inside the library, rather this approach reconstructs buildings by aggregating individually derived roof planes and/or building parts from the input point cloud data. Series operations of differing complexity need to be performed to find the topologies and relationships among the extracted planar segments, boundary segments and vertices. Effective use of the data-driven approaches can reconstruct any polyhedral building in more detail without depending on the predefined building library. However, in the data-driven approaches, low point density degrades the performance due to the missing building parts (e.g., chimneys, dormers, and vents) [11] and the incorrect classification of building roof features (e.g., boundary, fold, and planar roof points) can highly affect the building reconstruction [3]. To get the benefit from both model and data-driven techniques, a few hybrid approaches can also be found in the literature [5].

The points from the initial raw aerial LiDAR input point cloud data when separated into the building set can contain five different classes of building points [13]. The first class is the points that belong to the roof area only. The majority of the points belong to this class. The four other classes are roof vertical surface points, façade points, terrain, and noisy points due to the neighbouring vegetation and birds [13]. Correct segmentation of the roof planes and establishing the relationship among the planes are the major challenge in data driven-based approach of building reconstruction. The reconstructed $3 \mathrm{D}$ building models must be followed by an accuracy estimation process to measure the reconstructed buildings' fidelity to the original or reference building. Several techniques have been proposed and used for this which includes both visual and geometric estimation processes [21, 22, 23]. The visual estimation techniques use several parameters, such as voting from human experts, fuzzy set theories, and the quality of estimated dominant directions $[23,24]$. The geometric accuracy estimation methods take the reference and reconstructed building models and calculate feature-based and LiDAR-based accuracy measures [21].

Finally, regarding the successes achieved for developing automatic algorithms that carry out automatically the segmentation/classification and modelling tasks simultaneously, several authors suggested global automatic algorithms that extract and model the buildings from LiDAR data $[10,38,39,34]$. These approaches suffered from several limitations due to the classification accuracy and the cases of building modelling failure.

\section{DATASET}

In this study, one dataset is used (see Fig. 1). The characteristics of this dataset are shown in Table I. The dataset, which was captured over the city of Vaihingen in Germany [29], contains residential buildings, historic buildings, and small-detached houses that are surrounded by trees. It is important to note that only the first returns of laser beams are employed in this study.

TABLE I. CHARACTERISTICS OF USED DATASETS

\begin{tabular}{|c|c|}
\hline & Vaihingen \\
\hline Acquisition & 21 August 2008 \\
\hline Sensor & $\begin{array}{c}\text { Leica Geosystems Leica } \\
\text { ALS50 system }\end{array}$ \\
\hline Point density & $6.7-4$ point $/ \mathrm{m}^{2}$ \\
\hline Flight height & $500 \mathrm{~m}$ \\
\hline
\end{tabular}

The following section presents the full classification and modelling algorithm. The algorithm input is the city LiDAR point cloud and its output is the city model.

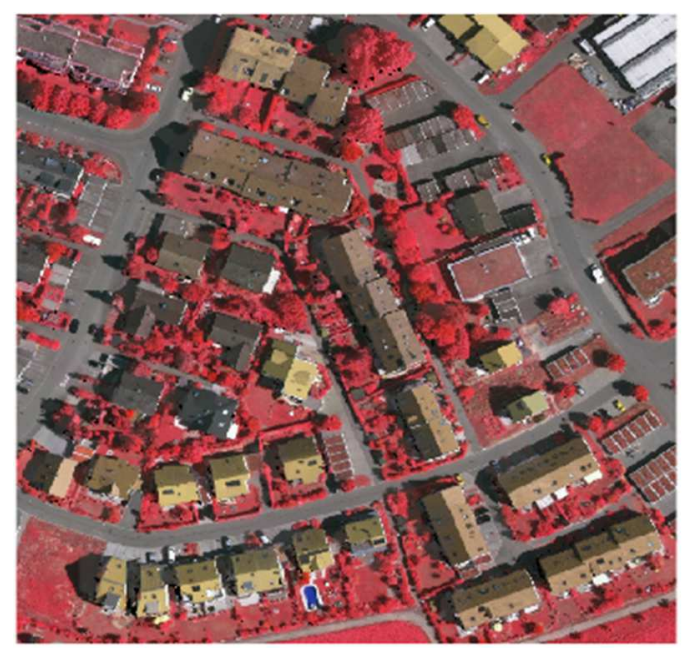

Fig 1. Aerial image of study area

\section{FULL CLASSIFICATION AND MODELLING ALGORITHM}

The algorithm illustrated in Fig. 2 represents a full classification and modelling algorithm that can link any classification and modelling algorithms that are suggested in 
the literature. The sequence of this algorithm may be summarized as follows:

- Application of the classification algorithm to classify the city LiDAR point cloud into three classes which are terrain, vegetation and buildings.

- Filtering of the building class by eliminating the trees associated with the building and eliminating the trees classified as buildings.

- Reassigning the misclassified building points to the building class.

- Application of the region growing algorithm on the building mask for detecting each building separately.

- Application of modelling algorithm on each building to construct the building model.

In modelling algorithm:

- After segmentation of the building roof, evaluation of the segmentation result.

- If the roof segmentation is successful, the building model is constructed.

- If the roof segmentation is not successful, modelling the building with a flat roof.

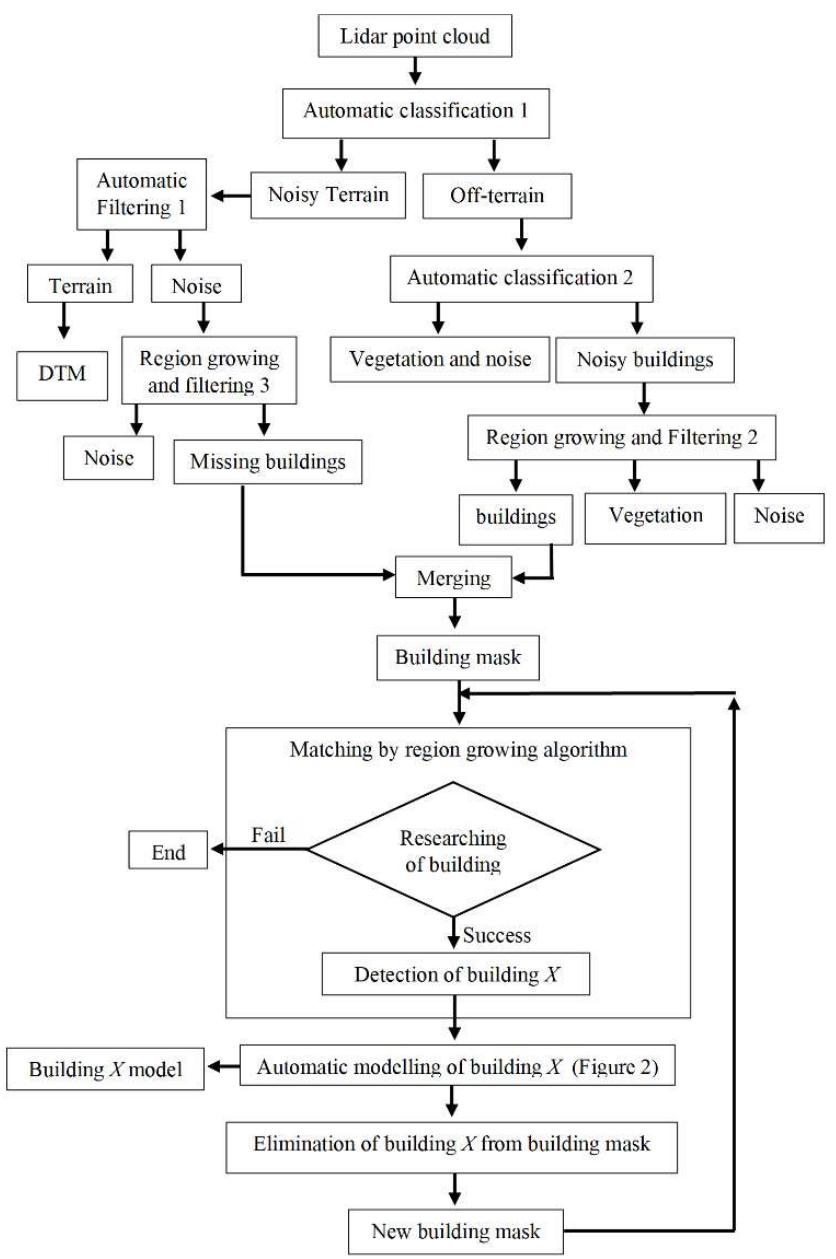

Fig 2. Flowchart of full classification and modelling algorithm

As shown above, the constructed city model contains two types of building models: buildings with modelled roofs and buildings with non-modelled roofs (in the case of roof segmentation failure). The roof modelling failures occur because of a result of the architectural complexity of the building roof combined with low point density and altimetry accuracy.

In the next subsections, the main points of the modelling algorithm will be detailed.

\section{A. Pre-processing}

As shown in Fig. 2, the suggested algorithm consists of three main parts: the classification part, the classification and modelling linking part and the modelling part. In the first and the second parts of the suggested approach, vector and raster data forms will be employed together. Indeed, the use of the normalized Digital Surface Model (nDSM) will facilitate creating the link between the classification and segmentation algorithms.

To link the nDSM with the point cloud, the point cloud is superimposed over an empty matrix with the same dimensions as the last nDSM. Each cell in this matrix may contain a set of points that are located within the cell borders. This matrix is named Cloud 2D Matrix (C2DM), e.g., one non-zero pixel in $\mathrm{nDSM}$ corresponds to a list of points (belonging to the point cloud) that are projected in this pixel. Consequently, if a group of pixels of the nDSM is assigned to the building class, the list of points located in these pixels can be directly extracted from the point cloud and likewise assigned. In this context, the matrix C2DM offers the possibility of directly recognizing the set of points that are assigned to a given class.

\section{B. Application of classification algorithm}

In literature, there are two families of suggested building classification approaches for urban LiDAR point clouds. The first one achieves the classification into two steps which are: terrain / off-terrain classification and then the classification of off-terrain class into buildings and vegetation [6]. The second classification approach family extracts the building class directly from the city point cloud [17]. In this paper, the first classification family was used. In Fig. 2, automatic classification 1 refers to the terrain / off terrain classification. The result of this classification will be into two classes which are noisy terrain (Fig. 4) and off-terrain (Fig. 3b). Fig. 3a shows the Digital Surface Model (DSM) of the selected area calculated from the LiDAR point cloud. The off-terrain class represents most of buildings that have considerable heights and areas in addition to trees of comparable height. However, it may also contain noisy points, that are those that represent neither trees nor buildings or points that result from multiple reflections.

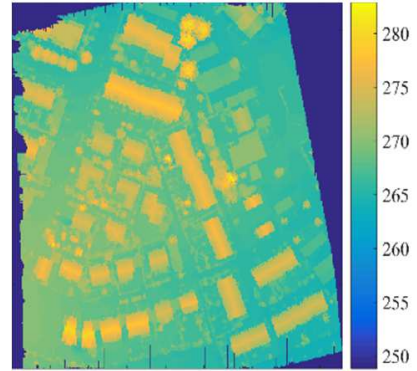

(a)

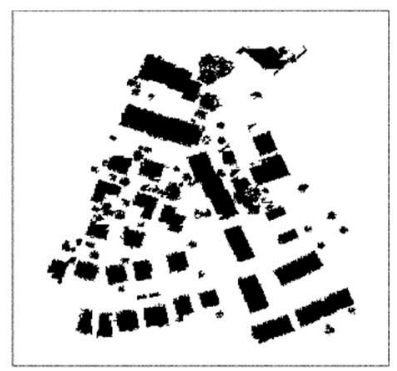

(b)
Fig3. (a) DSM of studied area; (b) Off-terrain mask 
The noisy terrain class represents the terrain, small vegetation, missing buildings and noise. According to Chen et al. [30], there are six main method categories for generating the Digital Terrain Model (DTM) from LiDAR data which are: surface-based adjustment, morphology-based filtering, Triangulated Irregular Network (TIN)-based refinement, segmentation and classification, statistical analysis and multiscale comparison. In our case, the use of the morphologybased filtering approach was suitable because the input data was the noisy terrain point cloud. In this context, the approach suggested by Susaki [31] can be employed.

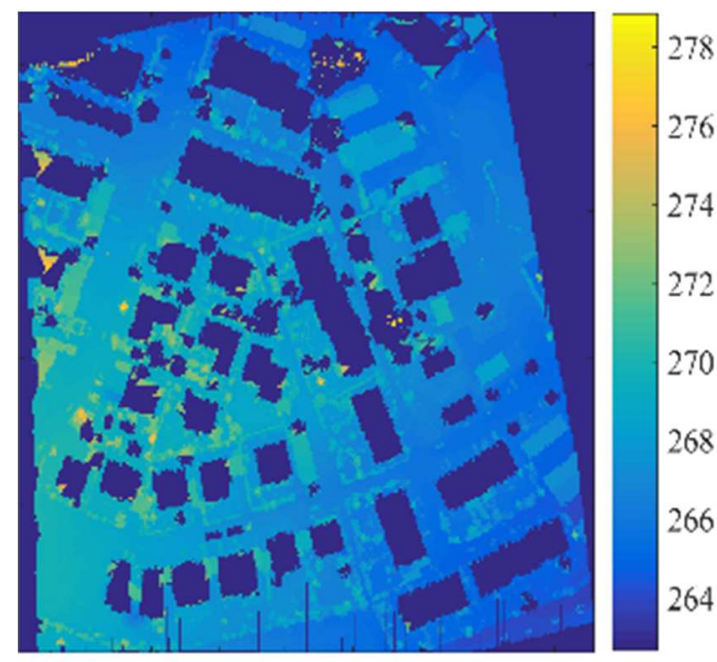

Fig 4. Noisy terrain class

Once the DTM is calculated, the noisy points from the noisy terrain class can be extracted and analysed to detect the missing building class (Fig. 6a). For this purpose, three main criteria can be employed: the segmented area, the geometric form and the standard deviation. The off-terrain point cloud is classified into vegetation and noisy building sub-classes. The noisy building sub-class (Fig. 5) contains the buildings detected among the off-terrain class in addition to noises that represent some high trees associated to the building roof (see red circles in Fig. 5) and some vegetation segments that have point distribution similar to building points.

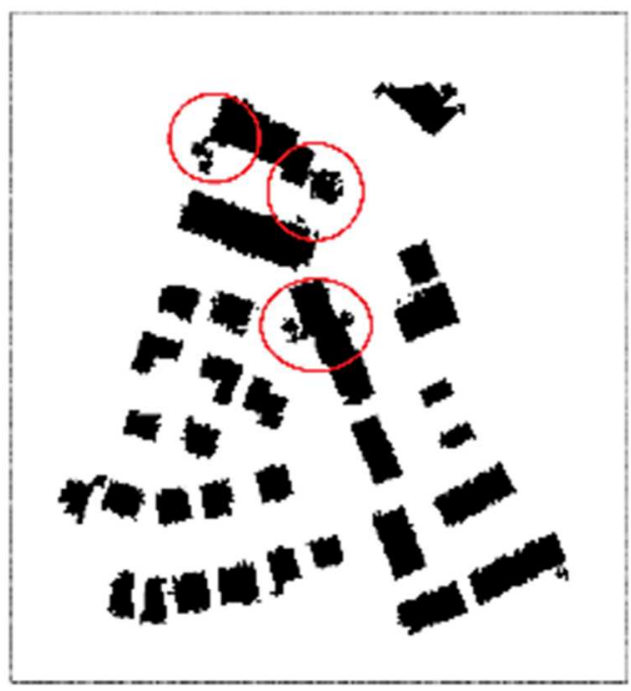

Fig 5. noisy building mask

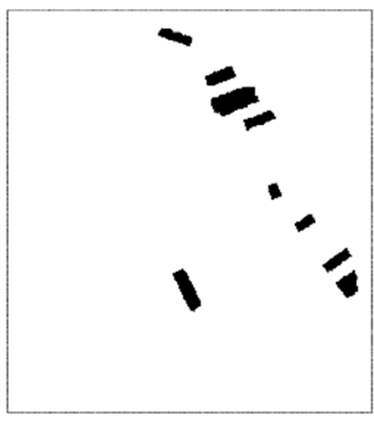

(a)

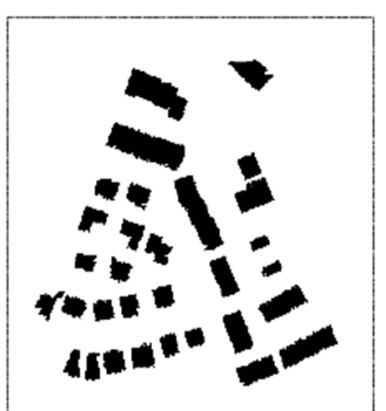

(b)
Fig 6. (a) Missing building mask; (b) Filtered building mask

Once the missing building mask and the noisy building mask are extracted, the next step is the filtering of the noisy building mask.

\section{Filtering of noisy building mask}

The input of this operation is the noisy building mask. To filter the noisy building mask, the approach suggested by Tarsha Kurdi et al. [32] was used. This choice has been adopted because of three advantages offered by this approach. First, as the modelling approaches used for constructing the building model use the hypothesis that the building point cloud consists of only the roof surfaces [33], the building cloud points need to be classified into two classes which are the roof points and the non-roof (undesirable) points. In fact, the undesirable points sometimes represent an obstacle to the modelling algorithms, especially when their percentage exceeds certain limits. Sometimes they are considered as the main reason for automatic modelling failure or the source for some deformations in the obtained building model [21]. Second, it considers an important case, which is the case of high trees associated with the building roof. Third, it can recognise the remaining vegetation segments that have a point distribution similar to building points.

This algorithm extracts the roof point cloud from the building point cloud by analysis of the $Z$ histogram of the building point cloud. Thereafter, three criteria are calculated and analysed: the normal vector, the standard deviation, and the change of curvature factor. At this stage, the employed threshold values are automatically determined for each building point cloud independently. This procedure increases the filtering success percentage because it considers the distinctive of the point cloud of each building. For more details about this algorithm please see Tarsha Kurdi et al. [32].

Fig. 8 shows the result of the application of this filter on the noisy building class.

\section{Calculation of final building mask}

At this stage of processing, there are now two building masks: the missing building mask and the filtered building mask. The final building mask is the result of the pixel-bypixel summation of the two nDSM of the two building masks (Fig. 8).

Once the final building mask is calculated, the region growing algorithm is applied on the nDSM of the final building mask to detect each building segment separately and then send it to the modelling algorithm for constructing its model. 


\section{E. Modelling algorithm}

Most of building modelling algorithms are based on hypotheses for constructing the building models [13], e.g., the model-driven approaches try to select the most appropriate building model among a limited building library, and most data-driven approaches suppose that the building roofs consist of planar facets. Moreover, the point density, heterogeneity of point distribution, accuracy, and the proportion of noisy point play an important role in the success of the modelling algorithm for constructing the building model [21]. Therefore, in any urban LiDAR point cloud, it is reasonable to expect some modelling failure cases. To construct a full series algorithm for automatic building extraction and modelling, the algorithm must recognise the failure cases and suggest an initial solution for them. Otherwise, the automatic building modelling loop will be broken down. At this stage, the algorithm suggested by Tarsha Kurdi and Awrangjeb [12] for modelling the missing roof details were adopted and simultaneously adapted to be used for this goal (Fig. 7).

To recognise the building modelling failure cases, a new hypothesis is adopted. If the building roof segmentation is successfully achieved, the building model can successfully be constructed, and vice versa. In this context, the Segmentation Accuracy and Quality (SAQ) factor is employed [12]. The SAQ factor (\%) expresses the rate of the number of whole pixels in the building error map matrix having values outside the interval $[-0.25 \mathrm{~m},+0.25 \mathrm{~m}]$ to the total number of building pixels, where the error map matrix is a matrix that represents the deviation of LIDAR roof points from their fitting surfaces. The quality of the building roof segmentation according to the SAQ factor can be estimated according to Table II.

TABLE II. QUALITY OF BUILDING ROOF SEGMENTATION ACCORDING TO SAQ FACTOR [12]

\begin{tabular}{|c|c|c|c|c|c|}
\hline SAQ (\%) & $>20$ & {$[20,15]$} & {$[10,15]$} & {$[5,10]$} & {$[0,5]$} \\
\hline $\begin{array}{c}\text { Accuracy } \\
\text { estimation }\end{array}$ & Rejected & Passable & Good & $\begin{array}{c}\text { Very } \\
\text { good }\end{array}$ & Excellent \\
\hline
\end{tabular}

Once the SAQ factor value (SAQ1 in Table IV) is smaller than $20 \%$ (Table II), the roof segmentation can be improved. For this purpose, every point having a noise value outside the interval $[-0.25,+0.25]$ must be projected on to its corresponding surface. This operation allows the generation of an improved roof point cloud. The next step is to resegment the entire improved point cloud of the roof and recalculate the SAQ factor (SAQ2 in Table IV). If the new SAQ factor value is smaller than its first value and also smaller than $8 \%$, then the roof segmentation is successful and the building model can be successfully constructed. Otherwise, if the SAQ factor value recommends the rejection of the roof segmentation, then the roof modelling is considered to have failed. In failed cases, the suggested algorithm proposes the construction of a building model with a flat roof, in other words, the suggested approach advises only to model using the roof outer boundaries.

In Fig. 7, it can be noted that the SAQ factor plays a double role during the modelling algorithm. First, it allows the identification of the modelling failure cases. Second, it permits an estimation of the possibility of modelling the missing roof details. For more details about the SAQ factor please see Tarsha Kurdi and Awrangjeb [12].

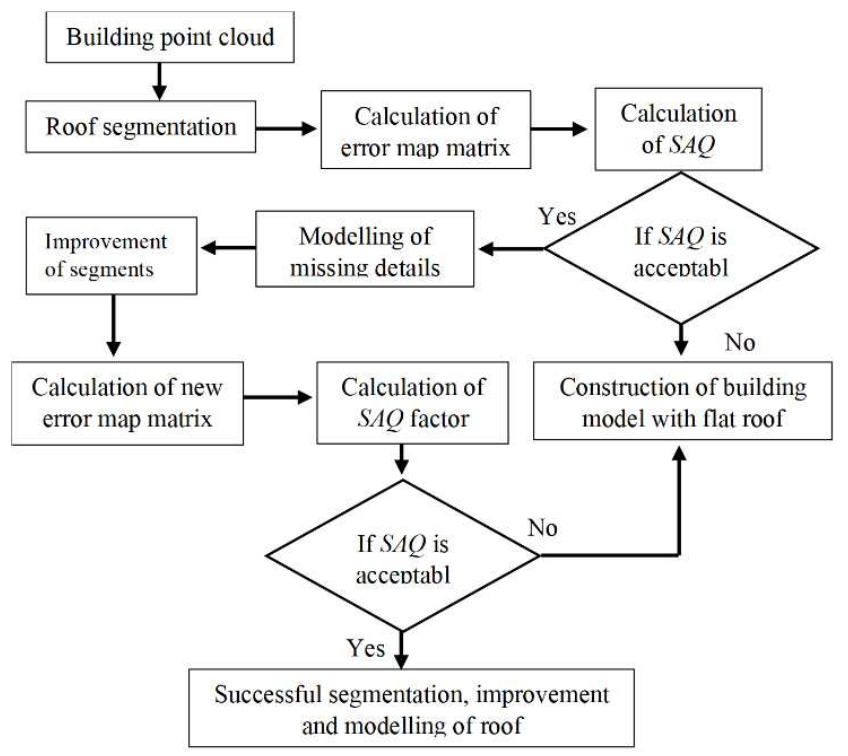

Fig 7. Automatic modelling of building $X$

\section{RESULTS}

One test site in the Vaihingen point cloud was selected. It contained 38 buildings. It is important to note that the classification approach employed in this study was suggested by Tarsha Kurdi et al. [37]. This technique carries out the classification into two consecutive steps. In the first step, terrain and off-terrain clouds are discriminated, based mainly on threshold values, and it considers the case of severe terrain discontinuities. In the second step, buildings and vegetation are categorized as subclasses of the off-terrain class through analysing the spatial location of the neighbouring points. Fig. $3 \mathrm{~b}$ illustrates the nDSM of the off-terrain class whereas Fig. 8 represents the nDSM of the final building mask.

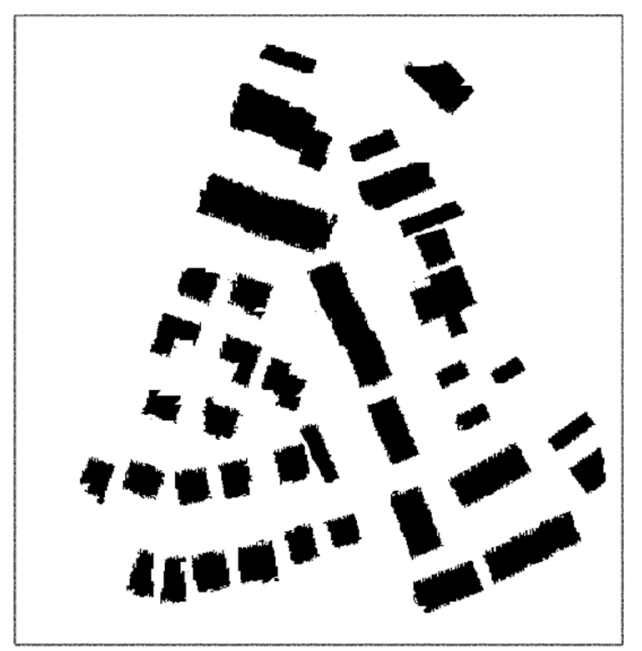

Fig 8. Final building mask

The modelling approach used is that which suggested by Tarsha Kurdi et al. [13]. It starts by detecting the roof planes and calculating their adjacency matrix. Hence, the roof plane boundaries are classified into four groups: outer boundary, inner plane boundaries, roof detail boundaries and boundaries related to the missing planes. The junction relationships of roof plane boundaries are analysed to detect the roof vertices. Fig. 9 presents the final result of a full series algorithm of 
automatic building classification and modelling for the test site. In Fig. 9, three colours are used, blue represents the building's outer boundaries, red represents the inner roof plane boundaries and green represents the missing roof details.

The quantitative analysis and the accuracy of these results are reported in the next section.

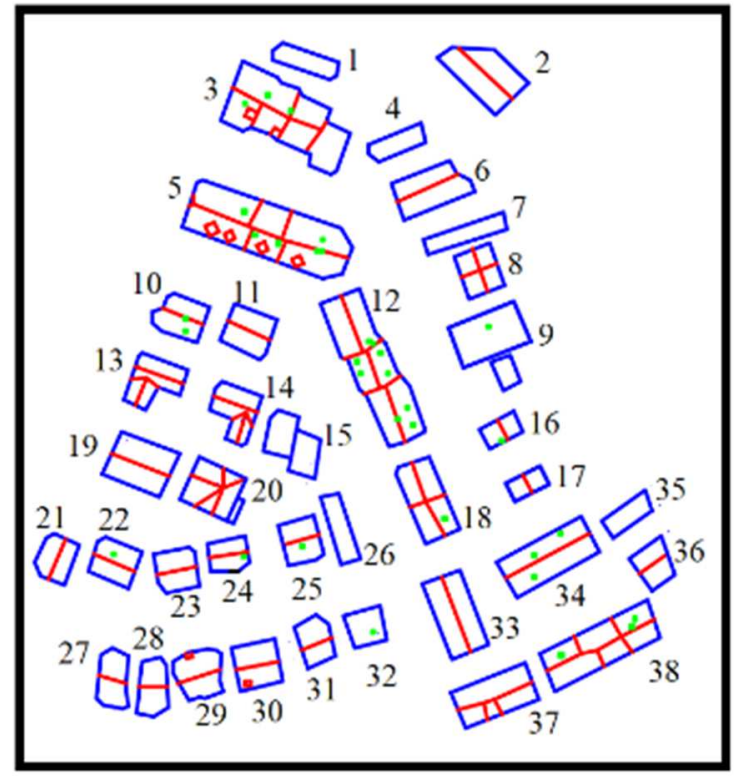

Fig 9. Final result of automatic 2D building modelling of Vaihingen site; blue: the building outer boundaries; red: the inner roof boundaries; green: missing details

\section{ACCURACY AND ANALYSIS}

In this paper, two accuracy analysis studies are used, the first one is of the first part of the algorithm which is the automatic building classification. The second one concerns automatic building modelling.

\section{A. Accuracy of classification algorithm}

To evaluate the accuracy of the building classification algorithm, the confusion matrix has been used [36]. For this purpose, two classes will be considered: the building class and the non-building class. The reference model is constructed by manual classification, which is assumed to have suitable accuracy [28]. Three errors may describe the classification result accuracy (Table III). Error I represents the rate of misclassified building pixels; Error II describes the rate of misclassified non-building pixels, and Total Error which expresses the rate of misclassified pixels.

TABLE III. BUILDING CLASSIFICATION ACCURACY

\begin{tabular}{|c|c|c|}
\hline Error I (\%) & Error II (\%) & Total Error (\%) \\
\hline 0.6 & 0.02 & 0.01 \\
\hline
\end{tabular}

Table III shows that despite the values of Error I and II being negligible, Error I is greater than the Error II, because the number of building class pixels in the nDSM is much smaller than the number of non-building class pixels. Furthermore, the presence of noise and non-building objects (such as trees) associated with the building roofs are the reason of the misclassification in the three error types. Indeed, even in the manual extraction, the presence of confusing points sometimes makes the operators not exactly sure of their decision. However, the minimum values of the committed errors in the classification step (thanking to the employed filtering algorithms) will have a negligible effect during the modelling procedures. The excellent result provided by the suggested algorithm is due to the consideration of missing buildings shows its utility.

\section{B. Accuracy of Modelling algorithm}

In literature, Tarsha Kurdi and Awrangjeb [21] summarize the geometric accuracy estimation approaches into two classes. On one hand, the feature-based estimation accuracy approach requires a reference model to compare the calculated model with the reference, e.g., Rottensteiner and Clode [34] produce the reference model in a semiautomatic working environment by using digital aerial images and LiDAR data. On the other hand, the LiDAR-based approach, the LiDAR point cloud is used as reference data, e.g., Erberink and Vossleman [35] employed the LiDAR point cloud as a reference model. Hence, the accuracy estimation approach employed in this paper belongs to the second class where the building point cloud is considered as reference data.

To estimate the accuracy of the $2 \mathrm{D}$ building models presented in Fig. 9, the SAQ factors were calculated before and after the roof cloud improvement (see Section IV-E and Table IV). In Table IV, it can be seen that the most of buildings have an excellent accuracy estimation with regards to the value of the SAQ factor after improvement (SAQ is smaller than 5\%) (See Table II). These results show the success of building roof segmentation and to the validity of the modelling of missing details in addition to the validity of the roof segments improvement.

TABLE IV. VALUES OF SAQ FACTOR BEFORE AND AFTER IMPROVEMENT OF FIRST VAIHINGEN SITE

\begin{tabular}{|c|c|c|c|c|c|c|c|}
\hline $\begin{array}{c}\text { Building } \\
\text { number }\end{array}$ & 1 & 2 & 3 & 4 & 5 & 6 & 7 \\
\hline SAQ1(\%) & 21.62 & 9.43 & 9.59 & 0.12 & 2.55 & 1.54 & 22.4 \\
\hline SAQ2(\%) & -- & 5.5 & 1.17 & -- & 0.84 & 0 & -- \\
\hline $\begin{array}{c}\text { Building } \\
\text { number }\end{array}$ & 8 & 9 & 10 & 11 & 12 & 13 & 14 \\
\hline SAQ1(\%) & 1.71 & 1.73 & 1.22 & 1.64 & 2.61 & 0.56 & 0.38 \\
\hline SAQ2(\%) & 1.65 & 0.44 & 0.25 & 0.35 & 0.83 & -- & -- \\
\hline $\begin{array}{c}\text { Building } \\
\text { number }\end{array}$ & 15 & 16 & 17 & 18 & 19 & 20 & 21 \\
\hline SAQ1(\%) & 5.3 & 0.22 & 0 & 2.64 & 1.01 & 0.89 & 0.88 \\
\hline SAQ2(\%) & 4.8 & -- & -- & 1.00 & 0.2 & -- & -- \\
\hline $\begin{array}{c}\text { Building } \\
\text { number }\end{array}$ & 22 & 23 & 24 & 25 & 26 & 27 & 28 \\
\hline SAQ1(\%) & 0,98 & 0.16 & 0.26 & 0.43 & 3.45 & 2.25 & 3.04 \\
\hline SAQ2(\%) & -- & -- & -- & -- & 2.91 & 1.29 & 0.79 \\
\hline $\begin{array}{c}\text { Building } \\
\text { number }\end{array}$ & 29 & 30 & 31 & 32 & 33 & 34 & 35 \\
\hline SAQ1(\%) & 1.36 & 2.15 & 9.75 & 1.68 & 6.4 & 0.94 & 6.28 \\
\hline SAQ2(\%) & 0.86 & 0.90 & 5.42 & 0.87 & 2.37 & -- & 4.09 \\
\hline $\begin{array}{c}\text { Building } \\
\text { number }\end{array}$ & 36 & 37 & 38 & & & & \\
\hline SAQ1(\%) & 0.92 & 0.14 & 3.53 & & & & \\
\hline SAQ2(\%) & -- & -- & 1.44 & & & & \\
\hline
\end{tabular}

In Table IV, the modelling of Building 1 failed (its SAQ factor was greater than 20\%) and the result of roof segmentation was rejected, which is why it was modelled with a flat roof. In Fig. 9, there are two categories of buildings that have been modelled with flat roofs: first, those that fail the modelling operation, e.g., the case of Building 1, second, the original roof of the building is flat, e.g., the case of Building 
9. Moreover, Buildings 4, 13, 14, 16, 17, 20, 21, 22, 23, 24, $25,34,36$ and 37 have very accurate results after the first roof segmentation as shown by their SAQ factors being smaller than $1 \%$ (see Table IV). Since they did not need to improve their roof segmentation, the SAQ factors were not recalculated.

\section{Comparison of proposed approach with existing methods}

At this stage, it is important to compare the functionality of the suggested algorithm with other existing ones [10, 38, $39,34]$. For this purpose, a descriptive and quantitative comparison are carried out.

In the descriptive comparison (Table V), the suggested approach is decomposed into its main functions, then the presence of these elements is checked in the existing algorithms. From Table V, each one of the existing algorithms misses some required functions that assure the algorithm efficacity. Indeed, the suggested approach tried to fill the most gaps which effect on the success and the accuracy of the result.

TABLE V. DESCRIPTIVE COMPARISON OF PROPOSED APPROACH WITH EXISTING METHODS

\begin{tabular}{|l|c|c|c|c|c|c|c|}
\hline \multicolumn{1}{|c|}{ Methods } & S1 & S2 & MB & AT & MA & AF & MD \\
\hline Suggested approach & Y & Y & Y & Y & Y & Y & Y \\
\hline Chen et al. [10] & Y & Y & N & N & Y & Y & N \\
\hline Forlani et al. [38] & Y & Y & N & Y & Y & N & N \\
\hline Rottensteiner [39] & Y & Y & N & N & Y & Y & N \\
\hline Rottensteiner [34] & Y & Y & Y & N & Y & Y & N \\
\hline
\end{tabular}

SA: Suggested Algorithm; S1: Segmentation Terrain / off-terrain; S2: Segmentation Building /

Vegetation; MB: Missing Building detection; AT: Associated Tree elimination; MA: Modelling

Algorithm; AF: Analysis of modelling Failure cases; MD: Missing roof Detail modelling; Y: Yes; N: No.

In the quantitative comparison (Tables VI and VII), the classification accuracy in addition to the modelling accuracy are compared between the suggested approach and the existing methods according to the availability of accuracy estimation results. On one hand, from Table VI, the high accuracy of the suggested approach (Error 0.6\%) may be explained by the elimination of the associated trees with building roofs in addition to the detection of the missing buildings. On the other hand, the great value of modelling completeness in the suggested approach is due to the high quality of the classification result in addition to the consideration of the missing roof details (see Table V). In fact, the missing roof details were sometimes considered in the existing approaches as undesirable noise because of their small areas in comparison to the point density. Then, their presence decreases the modelling accuracy.

TABLE VI. COMPARISON OF CLASSIFICATION ACCURACY

\begin{tabular}{|l|c|}
\hline \multicolumn{1}{|c|}{ Methods } & Error I (\%) \\
\hline Suggested approach & 0.6 \\
\hline Forlani et al. [38] & 3 \\
\hline Rottensteiner [34] & 8 \\
\hline
\end{tabular}

In this paper, the objective of the accuracy estimation is to validate the suggested classification and modelling algorithm. This showed, the suggested algorithm was able to extract the buildings and then model them. The modelling failure cases were marked red in Table IV, and they are modelled with a flat roof.
TABLE VII. COMPARISON OF MODELLING ACCURACY

\begin{tabular}{|l|c|}
\hline \multicolumn{1}{|c|}{ Methods } & Completeness (\%) \\
\hline Suggested approach & 98.5 \\
\hline Chen et al. [10] & $91-100$ \\
\hline Forlani et al. [38] & 70 \\
\hline
\end{tabular}

\section{CONCLUSION}

In this paper, the suggested approach constructs a full building classification and modelling algorithm of the urban LiDAR point cloud. The approach's success is based on solving the failure cases in both classification and modelling algorithms. In the classification algorithm, the missing buildings were extracted by filtering the noisy terrain class and analysing the obtained result. The detected buildings were filtered to eliminate the vegetation associated with building roofs. The majority of the undesirable points such as the surrounding terrain and façade points, which will not be employed in the modelling procedure, were removed.

In the modelling algorithm, the employment of the SAQ factor enables the recognition the failed modelling cases. For these models with flat roofs are temporarily constructed for these cases. Accurate models for the failed building cases can be later constructed by the user using a specialised modelling algorithm or manually.

The suggested algorithm can link any classification and modelling algorithms as long as the classification algorithm consists of two steps: classification into terrain / off-terrain and then further separation of the off-terrain class into buildings and vegetation. Indeed, more investigation is required to adapt the suggested approach for most classification algorithms. Furthermore, the suggested algorithm must be tested with more data samples of different typologies and point densities.

\section{ACKNOWLEDGMENT}

The Vaihingen data set was provided by the German Society for Photogrammetry, Remote Sensing and Geoinformation (DGPF) [29]. http://www.ifp.unistuttgart.de/dgpf/DKEP-Allg.html.

\section{REFERENCES}

[1] A. Nurunnabi, G. West and D. Belton, "Outlier detection and robust normal-curvature estimation in mobile laser scanning 3D point cloud data," in Pattern Recognition, 48(4):1404\{1419, 2015.

[2] E. K. Dey, M. Awrangjeb and B. Stantic, "Outlier detection and robust plane fitting for building roof extraction from LiDAR data," in International Journal of Remote Sensing, 41(16), 6325-6354, 2020, https://doi.org/10.1080/01431161.2020.1737339.

[3] E. K. Dey, F. Tarsha Kurdi, M. Awrangjeb and B. Stantic, "Effective selection of variable point neighbourhood for feature point extraction from aerial building point cloud data," in Remote Sensing, 2021, 13,1520. https://doi.org/10.3390/rs13081520.

[4] M. Awrangjeb and C. S. Fraser, "Automatic segmentation of raw LiDAR data for extraction of building roofs," in Remote Sensing, 6(5), 2014, 3716-3751, https://doi.org/10.3390/rs6053716.

[5] M. Awrangjeb, S. A. N. Gilani and F. U. Siddiqui, "An effective datadriven method for 3 -d building roof reconstruction and robust change detection," in Remote Sensing, 2018, 10(10), 1512, https://doi.org/10.3390/rs10101512.

[6] Y. Park and J. M. Guldmann, "Creating 3D city models with building footprints and LIDAR point cloud classification: A machine learning approach," in Computers, environment and urban systems, 75, 76-89, 2019, doi: 10.1016/j.compenvurbsys.2019.01.004. 
[7] C. Stucker, A. Richard, J. D. Wegner and K. Schindler, "Supervised outlier detection in large-scale MVS point clouds for 3D city modeling applications," in ISPRS Annals of the Photogrammetry, Remote Sensing and Spatial Information Sciences, 4,2018, 263-270, doi:10.5194/isprs-annals-IV-2-263-2018.

[8] E. K. Dey, M. Awrangjeb and B. Stantic, "An unsupervised outlier detection method for 3D point cloud data," in IGARSS 2019-2019 IEEE International Geoscience and Remote Sensing Symposium (pp. 2495-2498) IEEE, doi: 10.1109/IGARSS.2019.8900118.

[9] S. A. N. Gilani, M. Awrangjeb and G. Lu, "Robust building roof segmentation using airborne point cloud data," in 2016 IEEE International Conference on Image Processing (ICIP) (pp. 859-863) IEEE, doi: 10.1109/ICIP.2016.7532479.

[10] D. Chen, L. Zhang, P. Mathiopoulos and X. Huang, "A methodology for automated segmentation and reconstruction of urban 3-D buildings from ALS point clouds," in IEEE Journal of Selected Topics in Applied Earth Observations and Remote Sensing 7 (10) 2014: 4199-4217. doi:10.1109/JSTARS.4609443.

[11] S. A. N. Gilani, M. Awrangjeb and G. Lu, "Segmentation of airborne point cloud data for automatic building roof extraction," in GIScience and Remote Sensing 55 (1), 2018: 63-89. doi:10.1080/17538947.2017.1395089.

[12] F. Tarsha Kurdi and M.Awrangjeb, "Automatic evaluation and improvement of roof segments for modelling missing details using LiDAR data," in International Journal of Remote Sensing, 41(12), 2020, 4702-4725, doi: 10.1080/01431161.2020.1723180.

[13] F. Tarsha Kurdi, M. Awrangjeb and Munir, N, "Automatic filtering and 2D modeling of airborne laser scanning building point cloud," in Transactions in GIS, 25(1), 2021, 164-188, doi: 10.1111/tgis.12685.

[14] X. Wang, Y. P. Luo, T. Jiang, H. Gong, S. Luo and X. W. Zhang, "A new classification method for LIDAR data based on unbalanced support vector machine," in 2011 International Symposium on Image and Data Fusion (pp. 1-4) IEEE, doi: 10.1109/ISIDF.2011.6024312.

[15] C. Becker, E. Rosinskaya, N. Häni, E. d'Angelo and C. Strecha, "Classification of aerial photogrammetric 3D point clouds," in Photogrammetric Engineering \& Remote Sensing, 84(5), 2018, 287295, doi: 10.14358/PERS.84.5.287.

[16] N. Lukač and B. Žalik, "GPU-based roofs' solar potential estimation using LiDAR data," in Computers \& Geosciences, 52, 2013, 34-41, https://doi.org/10.1016/j.cageo.2012.10.010.

[17] E. Maltezos, A. Doulamis, N. Doulamis and C. Ioannidis, "Building extraction from LiDAR data applying deep convolutional neural networks," in IEEE Geoscience and Remote Sensing Letters, 16(1), 2018, 155-159, doi: 10.1109/LGRS.2018.2867736.

[18] J. Huang, X. Zhang, Q. Xin, Y. Sun and P. Zhang, “Automatic building extraction from high-resolution aerial images and LiDAR data using gated residual refinement network," in ISPRS journal of photogrammetry and remote sensing, 151, 2019, 91-105, doi: 10.1016/j.isprsjprs.2019.02.019.

[19] J. Yuan, "Learning building extraction in aerial scenes with convolutional networks," in IEEE transactions on pattern analysis and

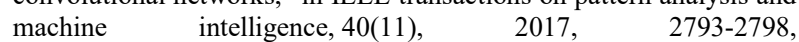
doi: 10.1109/TPAMI.2017.2750680.

[20] C. Wen, L. Yang, X. Li, L. Peng and T. Chi, "Directionally constrained fully convolutional neural network for airborne LiDAR point cloud classification," in ISPRS Journal of Photogrammetry and Remote Sensing, 162, 2020, 50-62, https://doi.org/10.1016/j.isprsjprs.2020.02.004.

[21] F. Tarsha Kurdi and M. Awrangjeb, "Comparison of LiDAR building point cloud with reference model for deep comprehension of cloud structure," in Canadian Journal of Remote Sensing, 46(5), 2020, 603621, https://doi.org/10.1080/07038992.2020.1829462.

[22] W. Ostrowski, M. Pilarska, J. Charyton and K. Bakuła, "Analysis of 3D building models accuracy based on the airborne laser scanning point clouds," in International Archives of the Photogrammetry, Remote
Sensing \& Spatial Information Sciences, 42(2), 2018, https://doi.org/10.5194/isprs-archives-XLII-2-797-2018, 2018.

[23] K. Zhang, J. Yan and S. C. Chen, A framework for automated construction of building models from airborne LIDAR measurements, in Topographic Laser Ranging and Scanning (pp. 511-534), 2017, CRC Press, doi: 10.1201/9781420051438.ch18.

[24] L. Cheng, W. Zhang, L. Zhong, P. Du and M. Li, "Framework for evaluating visual and geometric quality of three-dimensional models," in IEEE journal of selected topics in applied earth observations and remote sensing, 8(3), 2014, 1281-1294, doi: 10.1109/JSTARS.2014.2370753.

[25] D. Mongus and B. Žalik, "Efficient method for lossless LIDAR data compression," in International Journal of Remote Sensing, 32:9, 2011,2507-2518, doi: 10.1080/01431161003698385.

[26] M. Isenburg, "LASzip: lossless compression of LiDAR data," in Photogrammetric Engineering \& Remote Sensing 79(2), 2013, doi: 10.14358/PERS.79.2.209.

[27] A. Habib, Accuracy, quality, assurance and quality control of light detection and ranging mapping, in Topographic Laser Ranging and Scanning: Principles and Processing, edited by J. Shan, and C.K. Toth, 2nd ed., 2018, pp. 291-313. Boca Raton, FL: Taylor \& Francis Group; CRC Press.

[28] J. Shan and C.K. Toth, Topographic Laser Ranging and Scanning: Principles and Processing, 2nd ed., 2018, Boca Raton, FL: Taylor \& Francis Group; CRC Press.

[29] M. Cramer, "The DGPF test on digital aerial camera evaluation overview and test design," in Photogrammetrie - Fernerkundung Geoinformation 2(2010):73-82.

[30] Z. Chen, B. Gao and B. Devereux, " State-of-the-Art: DTM Generation Using Airborne LIDAR Data," in Sensors 2017, 17, 150; doi:10.3390/s17010150.

[31] J. Susaki, "Adaptive Slope Filtering of Airborne LiDAR Data in Urban Areas for Digital Terrain Model (DTM) Generation," in Remote Sens. 2012, 4, 1804-1819, doi: 10.3390/rs4061804.

[32] F. Tarsha Kurdi, M. Awrangjeb, E.K. Dey, Z. Gharineiat and G. Campbell, "Automatic filtering of LiDAR building point cloud, case of trees associated to building roof," unpublished.

[33] Y. He, Automated 3D building modelling from airborne Lidar data, $\mathrm{Ph}$.D. thesis, 2015, University of Melbourne, Australia.

[34] F. Rottensteiner and S. Clode, Building and road extraction from Lidar data, in: Shan, J. and Toth, C.K. 2ndEd, Topographic Laser Ranging and Scanning: Principles and Processing. CRC Press: Boca Raton, Taylor \& Francis Group, FL, 2018, pp. 485-522.

[35] S.O. Elberink and G. Vosselman, "Quality analysis on 3D building models reconstructed from airborne laser scanning data," in ISPRS Journal of Photogrammetry and Remote Sensing, 66(2), 2011, pp.157165. http://doi.org/10.1016/j.isprsjprs.2010.09.009.

[36] G. Sithole and G. Vosselmann, "Automatic Structure Detection in a Point-Cloud of an Urban Landscape," in 2nd Joint Workshop on Remote Sensing and Data Fusion over Urban Areas (URBAN2003), May 22-23, Berlin, Germany.

[37] F. Tarsha Kurdi, T. Landes, P. Grussenmeyer and E. Smigiel, "New approach for automatic detection of buildings in airborne laser scanner data using first echo only" in ISPRS Comm. III Symposium, Photogrammetric Computer Vision, Bonn, Sept. 20-22, 2006, Germany. International Archives of Photogrammetry and Remote Sensing and Spatial Information Sciences, ISSN: 1682-1750, Vol. XXXVI, Part 3, pp. 25-30.

[38] G. Forlani, C. Nardinocchi, M. Scaioni, and P. Zingaretti, "Complete classification of raw LIDAR data and 3D reconstruction of buildings," in Pattern Anal Applic 8, 357-374, 2006. https://doi.org/10.1007/s10044-005-0018-2.

[39] F. Rottensteiner and C. Briese, "A new method for building extraction in urban areas from high-resolution LIDAR data," in Int Arch Photogramm Remote Sens Spat Inf Sci 34(3A):2002, 295-301. 